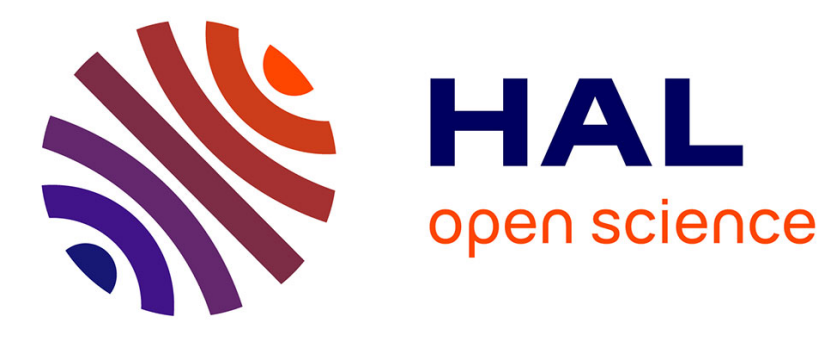

\title{
To Approximate Treewidth, Use Treelength!
}

David Coudert, Guillaume Ducoffe, Nicolas Nisse

\section{To cite this version:}

David Coudert, Guillaume Ducoffe, Nicolas Nisse. To Approximate Treewidth, Use Treelength!. SIAM Journal on Discrete Mathematics, 2016, 30 (3), pp.13. 10.1137/15M1034039 . hal-01348965

\section{HAL Id: hal-01348965 \\ https://inria.hal.science/hal-01348965}

Submitted on 26 Jul 2016

HAL is a multi-disciplinary open access archive for the deposit and dissemination of scientific research documents, whether they are published or not. The documents may come from teaching and research institutions in France or abroad, or from public or private research centers.
L'archive ouverte pluridisciplinaire HAL, est destinée au dépôt et à la diffusion de documents scientifiques de niveau recherche, publiés ou non, émanant des établissements d'enseignement et de recherche français ou étrangers, des laboratoires publics ou privés. 


\title{
TO APPROXIMATE TREEWIDTH, USE TREELENGTH!*
}

\author{
DAVID COUDERT ${ }^{\dagger \ddagger}$ AND GUILLAUME DUCOFFE ${ }^{\dagger}$ AND NICOLAS NISSE ${ }^{\dagger \ddagger}$
}

\begin{abstract}
Tree-likeness parameters have proven their utility in the design of efficient algorithms on graphs. In this paper, we relate the structural tree-likeness of graphs with their metric treelikeness. To this end, we establish new upper-bounds on the diameter of minimal separators in graphs. We prove that in any graph $G$, the diameter of any minimal separator $S$ in $G$ is at most $\lfloor\ell(G) / 2\rfloor \cdot(|S|-1)$, with $\ell(G)$ the length of a longest isometric cycle in $G$. Our result relies on algebraic methods and on the cycle basis of graphs. We improve our bound for the graphs admitting a distance preserving elimination ordering, for which we prove that any minimal separator $S$ has diameter at most $2 \cdot(|S|-1)$.

We use our results to prove that the treelength $t l(G)$ of any graph $G$ is at most $\lfloor\ell(G) / 2\rfloor$ times its treewidth $t w(G)$. In addition, we prove that, for any graph $G$ that excludes an apex graph $H$ as a minor, $t w(G) \leq c_{H} \cdot t l(G)$ for some constant $c_{H}$ only depending on $H$. We refine this constant when $G$ has bounded genus. Altogether, we obtain a simple $\mathcal{O}(\ell(G))$-approximation algorithm for computing the treewidth of $n$-node apex-minor-free graphs in $\mathcal{O}\left(n^{2}\right)$-time.
\end{abstract}

Key words. Graph; Treewidth; Treelength; Cycle basis; Genus.

AMS subject classifications. 05C85, 68Q17, 68Q25, 68R10

1. Introduction. It turns out that for a vast range of graph problems, the boundary between tractable and intractable cases depends on the tree-like properties of the graphs. This motivates us to study two tree-likeness invariants, that are called the treewidth [31] and the treelength [20]. Informally, the width of a tree-decomposition is the maximum size of its bags and its length is the maximum "diameter" of its bags. The treewidth and treelength of a graph are respectively the minimum width and length of its tree-decompositions (formal definitions can be found in Section 2). Note that bounded treelength graphs generalize the chordal graphs, split graphs, etc. which are well studied graph classes that have unbounded tree-width.

The treewidth aims to measure how close is the structure of a graph from the structure of a tree, whereas the treelength aims to measure the minimum distortion of the distances in a graph when it is embedded into a tree [1]. Since both invariants provide distinct, yet complementary, pieces of information on the closeness of a graph to a tree, we wish to relate treewidth and treelength through other graph properties so as to obtain a unifying view of tree-likeness in graphs.

Let us further motivate the need to compare treewidth with treelength, before presenting our main results.

One of the motivations is that we want to take the algorithmic advantages from both sides. Indeed, on the one hand there are many NP-hard problems that can be solved in polynomial-time on bounded-treewidth graphs [13]; on the other hand there exist compact routing schemes [7], approximation algorithms for packing, covering, and augmentation problems [10] up to an additive constant, as well as a PTAS for the well-known Traveling Salesman Problem on bounded-treelength graphs [26]. Therefore, finding relations between both invariants might lead to extend the use of some of the above-mentioned algorithms to a larger class of graphs. In particular,

*This work has been partially supported by ANR project Stint under reference ANR-13-BS020007, ANR program "Investments for the Future" under reference ANR-11-LABX-0031-01, and the Inria associated team AlDyNet.

${ }^{\dagger}$ Inria, France

${ }^{\ddagger}$ Univ. Nice Sophia Antipolis, CNRS, I3S, UMR 7271, 06900 Sophia Antipolis, France. 
this might be beneficial to bounded-treelength graphs that are more common than bounded-treewidth graphs amongst the complex networks, but for which there seem to be less algorithmic applications. For instance, the graph of Autonomous Systems has large treewidth, while it is hyperbolic $[1,14]$, and so, it has bounded treelength [9].

Another advantage of relating treewidth and treelength is that such relations can improve the best-known complexities for their computation on certain graph classes. Indeed, despite the fact that treewidth and treelength are both NP-hard to compute $[3$, 28], treelength seems much easier to approximate than treewidth. Namely, there are 3approximation algorithms for treelength that rely on a few breadth-first-searches [20], while the best known approximation algorithms for treewidth only achieve a ratio $\mathcal{O}(\sqrt{\log t w(G)})$ for general graphs - and a constant-ratio for minor-free graphsthrough the use of semi-definite programming [22]. We thus highlight that by relating treelength with treewidth, one can obtain practical algorithms for approximating the treewidth on certain graph classes.

1.1. State of the art. To put our contributions in context, let it be said that treewidth and treelength are uncomparable in general. This fact prevents from expecting simple relations between both invariants.

On the one hand, it comes from the fact that the cycle $C_{n}$, with $n \geq 4$ vertices, satisfies that $t w\left(C_{n}\right)=2$ while $t l\left(C_{n}\right)=\lceil n / 3\rfloor$. This suggests that having a large treelength relies on the existence of long cycles in the graph. The authors in [20] supported this intuition, proving that the treelength of a graph $G$ is bounded from above by half of the maximum length of a chordless cycle in $G$. Yet it is a strong constraint, as seen with the case of the wheel $W_{n}$ which contains an induced $C_{n}$ while it has treelength $\leq 2$. Therefore, it is natural to constrain ourselves to the subcase of isometric cycles in graphs. We remind that a subgraph $H$ of $G$ is isometric if for any two vertices of $H$, the distance between them is the same in $H$ as in $G$. Note that it is known how to compute a longest isometric cycle in a graph in polynomial-time [27]. Unfortunately, there are graphs such as grids with bounded-length isometric cycles and arbitrarily large treelength. As we will show below, our results imply that in such a case, we always have that $t l(G)=\mathcal{O}(t w(G))$.

On the other hand, the complete graph with $n$ vertices has treewidth $n-1$ and treelength 1. Another interesting example is the graph $H$ obtained by adding a universal vertex to a square-grid with $n^{2}$ vertices, for which it holds $t w(H)=n+1$ and $t l(H)=2$. Note that such graphs have a large genus, i.e., they are in a sense arbitrarily far from planar graphs. In contrast, it holds that $t w(G)<12 \cdot t l(G)$ for planar graphs [17]. Consequently, it is quite natural to ask whether a treewidth arbitrarily larger than the treelength requires a large genus. We will prove it is the case, i.e., $t w(G)=\mathcal{O}(t l(G))$ for bounded-genus graphs.

Finally, and independently from this work, Belmonte et al. [5] proved that $t w(G)=$ $\mathcal{O}\left(\Delta^{t l(G)}\right)$ for any graph $G$ with maximum degree $\Delta$. They built upon this relation in order to design a fixed-parameter-tractable algorithm to compute the metric dimension on bounded treelength graphs. We will use different techniques in order to upper-bound the treewidth with linear dependency on the treelength.

1.2. Our contributions. We introduce a very generic method to upper-bound the diameter ${ }^{1}$ of minimal separators in graphs, the latter denoting inclusion wise minimal subsets whose removal disconnects some fixed pair of vertices. Let us emphasize

\footnotetext{
${ }^{1}$ The diameter of a set $S$ of vertices of a graph $G$ is the maximum distance in $G$ between two vertices in $S$.
} 
that the minimal separators are at the cornerstone of various graph decompositions, such as the so-called $k$-connected decompositions [24]. Therefore, our method may find applications beyond the scope of tree-decompositions.

In a few more details, we prove that minimal separators in a graph $G$ induce connected subsets in some of its powers $G^{j}$, where $j$ only depends on the length of cycles in some arbitrary cycle basis of $G$ (see Section 2 for a formal definition). We deduce from our method that, for any graph $G$ with longest isometric cycle of size $\ell(G)$, and for any minimal separator $S$ in $G$, the diameter of $S$ is at most $\lfloor\ell(G) / 2\rfloor \cdot(|S|-1)$ and the upper-bound is sharp as shown by any cycle.

Then, we prove that for any graph $G$ which is not a tree, $t l(G) \leq\lfloor\ell(G) / 2\rfloor \cdot(t w(G)-1)$. This upper-bound on treelength follows from our upper-bound on the diameter of minimal separators, and it is tight up to a small constant-factor ${ }^{2}$. We refine our bound in several particular graph classes (the formal definition of these classes are postponed to the technical sections of the paper).

- For any graph $G$ in the class of null-homotopic graphs (including the class of dismantlable graphs), we prove that $t l(G) \leq t w(G)$. This is tight, as one can easily see on trees.

- In the class of graphs $G$ that admit a distance preserving elimination ordering, we prove that $t l(G)<2 \cdot t w(G)$.

We emphasize that the latter class contains the cobipartite graphs. Though the treelength of cobipartite graphs is trivially bounded by 3, computing their treewidth is an NP-hard problem. As a consequence, the treewidth of graphs that admit a distance preserving elimination ordering is also NP-hard to compute. Our results combined with the 3-approximation algorithm for treelength [20] provide a polynomial-time algorithm for computing a new non-trivial lower-bound for treewidth.

Finally, we consider lower-bounds for treelength. We prove that, for any graph excluding an apex graph $H$ as a minor, there is a constant $c_{H}$ such that $t w(G) \leq c_{H} \cdot t l(G)$. The constant $c_{H}$ only depends on $H$. Our proofs in this part make use of the bidimensionality theory [15]. In the particular case of graphs with bounded genus $g>0$, we use results from this theory so as to prove the more precise relation $t w(G) \leq 72 \sqrt{2}(g+1)^{\frac{3}{2}} \cdot t l(G)+\mathcal{O}\left(g^{2}\right)$.

So, to sum up, we obtain that any approximation algorithm for treelength can be turned into an approximation algorithm for treewidth up to multiplying by an $\mathcal{O}(\ell(G)$. $\left.(g+1)^{\frac{3}{2}}\right)$ the approximation ratio.

2. Preliminaries. In this section, we recall some useful definitions and known results that will be used in the sequel. All graphs considered in this paper are simple (i.e., without loops or multiple edges), connected and finite. Given a graph $G=$ $(V, E)$, the number $|V|$ of vertices will be denoted by $n$ and the number of edges $|E|$ by $m$. For any vertex $v \in V$, let $N_{G}(v)=\{u \in V \mid\{u, v\} \in E\}$ be the set of neighbors of $v$ in $G$. Let $N_{G}[v]$ denote $N_{G}(v) \cup\{v\}$.

Minimal separators. A set $S \subseteq V$ is a minimal separator if there exist $a, b \in V \backslash S$ such that any path from $a$ to $b$ intersects $S$ and, for any proper subset $S^{\prime} \subset S$, there is a path from $a$ to $b$ which does not intersect $S^{\prime}$. We name any such a set $S$ an $a-b$

\footnotetext{
${ }^{2}$ Recently and independently of this work (see research report in [12]), Diestel and Muller [19] proved that $t l(G) \leq \ell(G) \cdot(t w(G)-1)$ (see also [2] for a slightly looser bound). Note that our upper-bound for treelength is sharper than theirs. Furthermore, unlike our results which apply to any minimal separator in a graph, theirs rely on minimal separators in a specific tree-decomposition called an atomic tree-decomposition.
} 
minimal separator. A connected component $C \subseteq V \backslash S$ of $G[V \backslash S]$ is full with respect to $S$ if every node in $S$ has a neighbour in $C$. Any $a-b$ minimal separator has at least two full components: the one containing $a$ and the one containing $b$. Conversely, any separator having at least two full components is a minimal separator. A graph is said well-connected if each of its minimal separators induces a connected subgraph [21].

Cycle space. The set $\mathcal{C}(G)$ of Eulerian subgraphs of $G$ is called the cycle space of $G$. It is well-known that every Eulerian subgraph can be obtained from the symmetric difference (on the edges) of cycles in $G$. In fact, the set $\mathcal{C}(G)$ with the symmetric difference is a vector space of dimension $m-n+1$ if $G$ is connected[18, Theorem 1.9.6]. We will call the symmetric difference of two subgraphs $H_{1}, H_{2}$, denoted $H_{1} \oplus H_{2}$, the sum of $H_{1}$ with $H_{2}$. A cycle basis is an inclusion wise minimal set of cycles generating the whole cycle space In particular, a graph is said null-homotopic if it has a cycle basis with only triangles.

THEOREM 2.1. [21] Any connected null-homotopic graph is well-connected.

In this paper, we will extend the class of null-homotopic graphs as follows.

Definition 2.2. Let $l \geq 3$. We define $\mathcal{G}_{l}$ as the class of graphs whose cycle space can be generated by cycles of length at most $l$.

Note that $\mathcal{G}_{3}$ is exactly the class of null-homotopic graphs. Moreover, the isometric cycles in a graph can generate its cycle space [25] (see also Exercice 1.32 of the textbook [18]), so the class $\mathcal{G}_{l}$ contains all graphs with no isometric cycle longer than $l$. Therefore by varying the parameter $l$, classes $\mathcal{G}_{l}$ include all graphs and they form an inclusion wise increasing hierarchy. By [11], the smallest integer $l \geq 3$ such that a graph belongs to $\mathcal{G}_{l}$ can be computed in polynomial-time.

Diameter and Graph powers. For any $X \subseteq V$, let $\operatorname{diam}_{G}(X)$ denote the maximum distance in $G$ between any pair of vertices in $X$, a.k.a. the diameter of $X$. Last, for any $j \geq 1$, the graph $G^{j}$ is obtained from $G$ by adding an edge between any two distinct nodes that are at distance at most $j$ in $G$.

\subsection{Tree-decompositions.}

Minimal fill-in. A graph is chordal if all its induced cycles have length at most 3. For any graph $G=(V, E)$, we define a fill-in of $G$ as any chordal supergraph $H=(V, E \cup F)$ of $G^{3}$. A fill-in $H=(V, E \cup F)$ is minimal if, for any $f \in F$, the graph $H^{\prime}=(V, E \cup F \backslash\{f\})$ is not chordal.

Let $H$ be a fill-in of a graph $G$, and let $V_{C}$ be the set of maximal cliques of $H$. A cliquetree of $H$ is a tree $T_{C}=\left(V_{C}, F\right)$ such that for each vertex $x \in V$, the set of maximal cliques containing $x$ induces a subtree of $T_{C}$. We define a (reduced) tree-decomposition of $G$ as any clique-tree of an arbitrary fill-in of $G$. Equivalently, a tree-decomposition of $G$ consists of a pair $(T, \mathcal{X})$ where $T$ is a tree and $\mathcal{X}=\left(X_{t}\right)_{t \in V(T)}$ is a family of subsets of $V$, called bags, indexed by the nodes of $T$ and that satisfies the following three properties.

1. $\bigcup_{t \in V(T)} X_{t}=V$;

2. for any $\{u, v\} \in E$, there is $t \in V(T)$ with $u, v \in X_{t}$;

3. for any $u \in V$, the set of bags containing $u$ induces a subtree of $T$.

Tree-likeness invariants. Given a graph $G$, the length of a tree-decomposition $(T, \mathcal{X})$ equals the maximum diameter in $G$ of its bags. The treelength of $G$, denoted by $t l(G)$, is the minimum length over all tree-decompositions of $G$. Equivalently, the treelength of $G$ is the smallest integer $j$ such that $G^{j}$ contains a fill-in of $G$ [28]. The width of $(T, \mathcal{X})$ equals the maximum size of its bags minus one. The treewidth

\footnotetext{
${ }^{3}$ Here we use the term fill-in to avoid confusion with planar triangulations.
} 
of $G$, denoted by $t w(G)$, is the minimum width over all tree-decompositions of $G$. Equivalently, the treewidth of $G$ is the minimum over all minimal fill-ins $H$ of $G$ of $\omega(H)-1$, where $\omega(H)$ is the clique-number of $H$ [6]. It can be checked that both invariants are contraction-closed i.e., the contraction of an edge in the graph cannot increase its treewidth nor its treelength. We will often use the fact that treewidth and treelength are contraction-closed invariants in the following.

Parallel minimal separators. Finally, let $S_{1}, S_{2}$ be two minimal separators in a graph $G$. The separator $S_{1}$ crosses $S_{2}$ if there are two components of $G \backslash S_{2}$ that $S_{1}$ intersects. If $S_{1}$ does not cross $S_{2}$, then $S_{1}$ is said to be parallel to $S_{2}$.

THEOREM 2.3. [30] $H$ is a minimal fill-in of the graph $G$ if and only if $H$ is obtained by completing ${ }^{4}$ all sets of a maximal set of pairwise parallel minimal separators in $G$.

3. Diameter of Minimal Separators in Graphs. In this section, we show the diameter of any minimal separator $S$ in a graph $G$ is $\mathcal{O}(\ell(G) \cdot|S|)$, where $\ell(G)$ is the length of a longest isometric cycle in $G$ (Theorem 3.4). We then strengthen our results in particular graph classes that are defined by the existence of some elimination ordering of their vertices.

Before going into the details of the proof, let us describe the main intuition behind it and the difficulties we had to face on. Let us consider a minimal separator $S$. If it is connected, then the result easily follows. Hence, we may assume $S$ consists of several connected components. The idea is to find a set of paths, each of length at most $\lfloor\ell(G) / 2\rfloor$, such that any of these paths connects two components and the subgraph induced by $S$ and these paths is connected. If we do so, the result easily follows. Hence, the main difficulty is to find such paths. For this purpose, let us consider a minimum-length cycle crossing two components of $S$ (such a cycle surely exists because there are at least two full components in $G \backslash S$ ). If this cycle is isometric, then the distance between the two components cannot exceed $\lfloor\ell(G) / 2\rfloor$. Otherwise, it means that there is a shortcut between two nodes of the cycle. However, this shortcut could intersect $S$ more than once which does not help our purpose. The key point is that, using the shortcut, the initial cycle can be viewed as the combination (symmetric difference) of two cycles. This kind of local view can be generalized to a global one using our main tool, namely the cycle basis. Indeed, the initial cycle is actually the symmetric difference of a set of isometric cycles [25, 18]. Using this set, we can then prove our theorem.

3.1. Case of general graphs. We start proving some properties of graphs in the class $\mathcal{G}_{l}$. This will lead us to the main result in this section (Theorem 3.4).

Let us first prove that the class $\mathcal{G}_{l}$ is stable under the following two operations.

Lemma 3.1. Let $l \geq 3$, the class $\mathcal{G}_{l}$ is stable under edge-contraction.

Proof. Let $G \in \mathcal{G}_{l}$ with $n$ vertices and $m$ edges. W.l.o.g., $G$ is connected. The dimension $\operatorname{dim}(\mathcal{C}(G))$ of the cycle space $\mathcal{C}(G)$ is $s=m-n+1$ ([18, Theorem 1.9.6]). Let $e \in E(G)$ such that $e$ lies on $k \geq 0$ triangles in $G$. By contracting $e$, we loose one vertex and $k+1$ edges, the edge $e$ and for each triangle which contains $e$ we have to remove one of the resulting multi-edges. Hence, $\operatorname{dim}(\mathcal{C}(G / e))=\operatorname{dim}(\mathcal{C}(G))-k$. Let $\left\{C_{1} \cdots, C_{s}\right\}$ be a basis of $\mathcal{C}(G)$ such that each $C_{i}$ has length at most $\ell$. Let $\left\{C_{1}^{\prime}, \cdots, C_{t}^{\prime}\right\}$ be the set of cycles in $G / e$ which are obtained by contracting $e$ on each $C_{i}$ and by removing triangles that contain $e$ from the list. Then $t \geq \operatorname{dim}(\mathcal{C}(G / e))=s-k$ (since at most $k$ triangles have been removed) and each $C_{i}^{\prime}$ has length at most $\ell$. We

\footnotetext{
${ }^{4}$ Completing a set of vertices is to make the set a clique.
} 
show that $C_{1}^{\prime}, \cdots, C_{t}^{\prime}$ are linearly independent in $\mathcal{C}(G / e)$, which proves that they form a basis of $\mathcal{C}(G / e)$. For purpose of contradiction, let us assume that $C_{i_{1}}^{\prime} \oplus \cdots \oplus C_{i_{r}}^{\prime}=0$ for $1 \leq i_{1}<\cdots<i_{r} \leq s, r>0$. Then $C_{i_{1}} \oplus \cdots \oplus C_{i_{r}}$ is either 0 or $e$. Therefore, the sum equals $e$ since the $C_{i_{j}}$ 's are linearly independent in $\mathcal{C}(G)$. This is a contradiction as $(V(G),\{e\})$ is not Eulerian.

Hence, since all cycles in the basis $\left\{C_{1}^{\prime}, \cdots, C_{t}^{\prime}\right\}$ have length at most $\ell$, it implies that $G / e \in \mathcal{G}_{l}$.

LEMma 3.2. Let $G_{1}$ and $G_{2}$ be two graphs such that $V\left(G_{1}\right) \cap V\left(G_{2}\right)=\{x, y\}$ and $E\left(G_{1}\right) \cap E\left(G_{2}\right)=\emptyset$, and let $G=G_{1} \cup G_{2}=\left(V\left(G_{1}\right) \cup V\left(G_{2}\right), E\left(G_{1}\right) \cup E\left(G_{2}\right)\right)$. If $G_{1}, G_{2} \in \mathcal{G}_{l}$ and $d_{G_{1}}(x, y)+d_{G_{2}}(x, y) \leq l$, then $G \in \mathcal{G}_{l}$.

Proof. Let $C$ be a cycle in $G$. We will prove that it is a sum of cycles of length at most $l$ in $G$. If it is a cycle in $G_{1}$ (resp. in $G_{2}$ ), then we are done as it is the sum of cycles of length at most $l$ by Definition 2.2. Else, it must contain the pair $x, y$ and it can be decomposed into: a $x y$-path in $G_{1}$, and a $x y$-path in $G_{2}$. Let $C_{l}$ be obtained from the union of a shortest $x y$-path in $G_{1}$ with a shortest $x y$-path in $G_{2}$. Note that $C_{l}$ has length $d_{G_{1}}(x, y)+d_{G_{2}}(x, y) \leq l$ by the hypothesis. Furthermore, $H=C \oplus C_{l}$ is an Eulerian subgraph of $G$. Let $H_{1}, H_{2}$ be the respective subgraphs of $H$ that are induced by the edges in $G_{1}, G_{2}$ (possibly empty). Note that $E\left(H_{1}\right) \cap E\left(H_{2}\right)=\emptyset$ by construction. We claim that both graphs $H_{1}, H_{2}$ are Eulerian subgraphs. Indeed, on the one hand the subsets $V\left(H_{1}\right) \backslash\{x, y\}, V\left(H_{2}\right) \backslash\{x, y\}$ are disjoint by the hypothesis and so, any vertex $\neq x, y$ in one of these graphs, say in $H_{1}$, has the same (even) degree in $H_{1}$ as in $H$. On the other hand, by construction each node amongst $x, y$ is incident exactly to one edge in $E(C) \cap E\left(G_{1}\right)$ (resp. in $E(C) \cap E\left(G_{2}\right)$ ) and to one edge in $E\left(C_{l}\right) \cap E\left(G_{1}\right)$ (resp. in $E\left(C_{l}\right) \cap E\left(G_{2}\right)$ ). As a result, nodes $x, y$ have degree either null or equal to 2 in $H_{1}$, and similarly they have degree either null or equal to 2 in $H_{2}$, which is even in both cases. Consequently, both $H_{1}, H_{2}$ are sums of cycles of length at most $l$ by the hypothesis because they are respective Eulerian subgraphs of $G_{1}, G_{2} \in \mathcal{G}_{l}$. Hence $H=H_{1} \oplus H_{2}$ is also a sum of cycles of length at most $l$ in $G$. This concludes the proof because $C=H \oplus C_{l}$.

Then, we prove that for any graph $G \in \mathcal{G}_{l}$, every minimal separator in $G$ must contain a pair of vertices that are at small distance to each other.

Lemma 3.3. Let $l \geq 3$, let $G \in \mathcal{G}_{l}$ and let $S$ be a minimal separator in $G$. Either $S$ is a cut-vertex, or there are two distinct nodes $x, y \in S$ such that $d_{G}(x, y) \leq\lfloor l / 2\rfloor$.

Proof. Suppose that $S$ does not consist of a single cut-vertex. If the subgraph induced by $S$ contains at least one edge $\{x, y\}$, then we are done as in such case $d_{G}(x, y)=1 \leq\lfloor\ell / 2\rfloor$. So, we assume that $S$ is a stable set. Let $A, B$ be two distinct full components of $G \backslash S$ and let $s, t \in S$ be two distinct vertices. By connectivity, there is an st-path $P$ whose internal vertices are contained in $A$, and in the same way there is a st-path $Q$ whose internal vertices are contained in $B$. Let $C$ be a cycle composed of $P$ and $Q$. Because $G \in \mathcal{G}_{l}$, there is some set $\mathcal{C}$ of cycles of length at most $\ell$ whose sum equals $C$. We claim that there is a cycle $C^{\prime} \in \mathcal{C}$ which intersects both $A$ and another component of $G \backslash S$. Otherwise, because $S$ is a stable set, the sum of all cycles that intersect $A$ must generate $P$. This is not possible, since it is not Eulerian. As $S$ separates the components, there are $x, y \in S \cap V\left(C^{\prime}\right)$ and so, since the length of $C^{\prime}$ is at most $\ell$, we deduce that $d_{G}(x, y) \leq\lfloor\ell / 2\rfloor$. $\square$

Finally, we can prove Theorem 3.4 and Corollary 3.5 below. Intuitively, we consider a pair of nodes $x, y \in S$, where $S$ is a minimal separator in some graph $G \in \mathcal{G}_{l}$. If $x, y$ are connected in the induced subgraph $G[S]$, then it is clear that their distance in $G$ is at most $|S|-1$. Else, we prove that there is a $x y$-path that intersects 
the connected components $C_{1}, C_{2}, \ldots, C_{k}$ of $G[S]$ consecutively, and that satisfies: $x \in C_{1}, y \in C_{k}$, and $\forall 1 \leq i<k$, there exists a cycle of length at most $l$ which intersects both $C_{i}$ and $C_{i+1}$. Every two consecutive components $C_{i}, C_{i+1}$ of $G[S]$ are thus at distance at most $\lfloor l / 2\rfloor$ in $G$, hence their union $C_{i} \cup C_{i+1}$ induces a connected subgraph of the power $G^{\lfloor l / 2\rfloor}$.

THEOREM 3.4. Let $l \geq 3$. For any graph $G \in \mathcal{G}_{l}$, every minimal separator in $G$ induces a connected subgraph in the power $G^{\lfloor l / 2\rfloor}$.

Proof. By contradiction, let $G \in \mathcal{G}_{l}$, and let $S$ be a minimal separator in $G$ that does not satisfy the property. We first make adjacent every two vertices in $S$ that are at distance at most $\lfloor l / 2\rfloor$ in $G$. We claim that the resulting graph is still in $\mathcal{G}_{l}$. Indeed, let $x, y \in S$ be non-adjacent and at distance at most $\lfloor l / 2\rfloor$ in $G$, let $G_{1}=G$ and let $G_{2}$ be the complete graph on the two vertices $x, y$ (i.e., $G_{2}$ is isomorphic to $K_{2}$ ). Since we have that $G_{1} \in \mathcal{G}_{l}$ by the hypothesis, that $G_{2} \in \mathcal{G}_{3} \subseteq \mathcal{G}_{l}$ and that $d_{G_{1}}(x, y)+d_{G_{2}}(x, y) \leq\lfloor l / 2\rfloor+1 \leq l$, then we deduce from Lemma 3.2 that $G_{1} \cup G_{2} \in \mathcal{G}_{l}$. The same argument can be applied iteratively because adding an edge in $G$ cannot increase the distances between nodes in $S$. So, the claim is proved. Finally, we contract each connected component of the subgraph induced by $S$ in a single node, thus contracting $S$ to obtain a stable set $S^{\prime}$, and the resulting graph $G^{\prime}$ still belongs to $\mathcal{G}_{l}$ by Lemma 3.1. Furthermore, the stable set $S^{\prime}$ is a minimal separator in $G^{\prime}$ by construction. Since $S$ does not satisfy the property of the theorem, we have that all nodes in $S^{\prime}$ are pairwise at distance at least $\lfloor l / 2\rfloor+1$, but then it contradicts Lemma 3.3.

Theorem 3.4 is tight, as it can be shown with any cycle $C_{l}$.

Corollary 3.5. Let $G$ be a graph that is not a tree. Any minimal separator $S$ in $G$ has diameter at most $\lfloor\ell(G) / 2\rfloor \cdot(|S|-1)$, where $\ell(G)$ denotes the length of a longest isometric cycle in $G$.

Proof. It follows from Theorem 3.4 combined with the fact that isometric cycles generate the cycle space $[25,18]$.

3.2. Graphs with distance-preserving elimination ordering. We strengthen the result of Corollary 3.5 in the case of graphs with a distance-preserving elimination ordering. Formally, we say that $G$ admits a distance-preserving elimination ordering if there exists a total order of $V$, denoted by $v_{1}, v_{2}, \ldots, v_{n}$, such that, for any $1 \leq i \leq n$, the subgraph $G_{i}=G \backslash\left\{v_{1}, \ldots, v_{i}\right\}$ is isometric. Graphs with a distance-preserving elimination ordering arise from applications in graph searching (e.g., dismantlable graphs [29]) and geometry [8]. Note that they contain the class of cobipartite graphs, for which computing the treewidth is NP-hard. Our main result in this section is that for every graph $G$ with a distance-preserving elimination ordering, it holds that $G \in \mathcal{G}_{4}$.

Proposition 3.6. A graph $G$ that admits a distance-preserving elimination ordering has its cycle space generated by all its triangles and quadrangles.

Proof. We claim that it is enough to prove that the induced cycles of $G$ can be generated by all its triangles and quadrangles. Indeed, the induced cycles of $G$ generate its cycle space [18]. Let $v_{1}, v_{2}, \ldots, v_{n}$ be a distance-preserving elimination ordering of $G$. By contradiction, amongst all induced cycles that do not satisfy the property let $C$ maximize the smallest index $j$ such that $v_{j} \in C$. Note that $C$ is a cycle of $G_{j-1}=G\left[\left\{v_{j}, \cdots, v_{n}\right\}\right]$ by the hypothesis. Moreover, all cycles contained in $G_{j}$ are the sum of triangles and quadrangles of $G$ because of the maximality of index $j$. Let $x, y \in V(C)$ be the two neighbours of $v_{j}$ in cycle $C$. By the hypothesis, $x, y$ are not adjacent because $C$ is induced. So, because $x, y, v_{j} \in G_{j-1}$ which has a distance- 
preserving elimination ordering, there is $v_{i}, i>j$ such that $x, y$ are adjacent to $v_{i}$. Moreover, $v_{i} \notin C$ because otherwise $C$ would be the quadrangle $\left(v_{j}, x, v_{i}, y, v_{j}\right)$, thus contradicting the fact that it does not satisfy the property. As a result, $C=Q \oplus C^{\prime}$, with $Q$ the quadrangle $\left(v_{j}, x, v_{i}, y, v_{j}\right)$ and $C^{\prime}$ is the cycle of $G_{j}$ obtained from $C$ by replacing the path $x, v_{j}, y$ with $x, v_{i}, y$. Furthermore, cycle $C^{\prime}$ is a sum of induced cycles of $G_{j}$ that are themselves a sum of triangles and quadrangles by maximality of $j$. Hence so is cycle $C$, which contradicts the fact that it does not satisfy the property. $\square$

COROLlaRY 3.7. Let $G$ be a graph that admits a distance-preserving elimination ordering. Every minimal separator $S$ in $G$ has diameter at most $2 \cdot(|S|-1)$.

Given that the cycle of length four $C_{4}$ admits a distance-preserving elimination ordering, one can see that Corollary 3.7 is sharp.

Dismantlable graphs are an interesting subclass of graphs with a distance-preserving elimination ordering. Formally, a graph $G$ is dismantlable if, for any $1 \leq i<n$, there exists $j>i$, such that $N_{G}\left[u_{i}\right] \backslash\left\{u_{1}, \cdots, u_{i-1}\right\} \subseteq N_{G}\left[u_{j}\right]$. It is immediate that if a graph is dismantlable, then it admits a distance-preserving elimination ordering. We obtain the following improvement over Proposition 3.6 for the subclass of dismantlable graphs.

Lemma 3.8. A dismantlable graph is null-homotopic and so, well-connected.

Proof. Let $G$ be a dismantlable graph. We prove that cycles of $G$ can be generated by its triangles, which proves that $G$ is null-homotopic. The fact that $G$ is wellconnected follows from the fact that dismantlable graphs are connected and from Theorem 2.1.

It is enough to prove that all induced cycles can be generated by triangles. Let $\left(u_{1}, u_{2}, \ldots, u_{n}\right)$ be a dismantling ordering of $G$. By contradiction, amongst all induced cycles that do not satisfy the property, let $C$ maximize the smallest index $j$ such that $u_{j} \in C$. Let $x, y \in V(C)$ be the two neighbours of $u_{j}$ in cycle $C$, and let $u_{i}$, with $i>j$, be a dominator of $u_{j}$ in $G_{j-1}$. We have that $u_{i} \notin C$ because $C$ is induced and it has length at least 4 by the hypothesis. As a result, $C=T_{1} \oplus T_{2} \oplus C^{\prime}$, with $T_{1}$ the triangle induced by nodes $u_{i}, x, u_{j}$; with $T_{2}$ the triangle induced by nodes $u_{i}, y, u_{j}$; and with $C^{\prime}$ a cycle of $G_{j}$ obtained from $C$ by replacing the path $x, u_{j}, y$ with $x, u_{i}, y$. Furthermore, cycle $C^{\prime}$ is a sum of induced cycles of $G_{j}$ that are themselves a sum of triangles of $G$ by maximality of $j$. Hence, so is cycle $C$, which contradicts the fact that it does not satisfy the property.

We note that it was already noticed in [21] that dismantlable graphs are nullhomotopic. However the proof was left to the reader. We give it in the paper for self-containment.

Corollary 3.9. Let $G$ be a dismantlable graph. Every minimal separator $S$ in $G$ has diameter at most $|S|-1$.

Last, we point out that by a result from [4], every graph is an isometric subgraph of some dismantlable graph. Therefore, there are graphs with arbitrarily long isometric cycles that admit a distance-preserving elimination ordering.

\section{Relating treewidth with treelength.}

4.1. Upper-bounds for treelength. Using the results recalled in Section 2.1, we are now able to upper-bound the treelength of a graph by a linear function depending on the size of its minimal separators. We then show that the treelength of a 
graph is upper-bounded by a function that is linear in its treewidth.

Lemma 4.1. Let $G$ be a graph and $\mathcal{S}$ be a maximal set of pairwise parallel minimal separators in $G$. If there is a constant $c_{\mathcal{S}}$ such that $\operatorname{diam}_{G}(S) \leq c_{\mathcal{S}}(|S|-1)$ for all $S \in \mathcal{S}$, then $t l(G) \leq \max \{1\} \cup\left\{c_{\mathcal{S}} \cdot(|S|-1) \mid S \in \mathcal{S}\right\}$.

Proof. Let $H$ be the supergraph of $G$ obtained by completing all sets of $\mathcal{S}$. By Theorem 2.3, $H$ is a minimal fill-in of $G$. Moreover, any clique-tree $T_{C}$ of $H$ corresponds to a reduced tree-decomposition of $G$ where each clique of $H$ induces a bag. Let $\Omega$ be any maximal clique in $H$, i.e., $\Omega$ is any bag of the tree-decomposition $T_{C}$. Let $x, y \in \Omega$. By definition of $H$, either $\{x, y\} \in E(G)$ or there is a minimal separator $S \in \mathcal{S}$ that contains both $x$ and $y$. In the latter case, $d(x, y) \leq \operatorname{diam}_{G}(S) \leq$ $c_{\mathcal{S}} \cdot(|S|-1) \cdot \mathrm{T}$

THEOREM 4.2. If every minimal separator in a graph $G$ induces a connected subgraph in its power $G^{j}$, then $t l(G) \leq \max \{1, j \cdot(t w(G)-1)\}$.

Proof. Let $H$ be a minimal fill-in of $G$ with maximum clique-size $t w(G)+1$. By Theorem 2.3, there is a maximal set $\mathcal{S}$ of pairwise parallel minimal separators of $G$ such that $H$ results from the completion of all elements in $\mathcal{S}$. Note that any $S \in \mathcal{S}$ induces a minimal separator in $H$ that is a clique - a.k.a. a clique-minimal separator in $H$ - and therefore $S$ is strictly contained in a maximal clique in $H$. Hence, $\max _{S \in \mathcal{S}}|S| \leq t w(G)$. By Lemma $4.1, t l(G) \leq \max \{1\} \cup\{j \cdot(|S|-1) \mid S \in$ $\mathcal{S}\} \leq \max \{1, j \cdot(t w(G)-1)\}$.

COROLlary 4.3. Let $G$ be a connected graph which is not a tree, then $\operatorname{tl}(G) \leq$ $c_{G} \cdot(t w(G)-1)$, where:

- $c_{G}=2$ if $G$ admits a distance-preserving elimination ordering;

- $c_{G}=\lfloor\ell(G) / 2\rfloor$, with $\ell(G)$ the length of a longest isometric cycle in $G$.

Proof. First item follows from Proposition 3.6 combined with Theorem 3.4 and Theorem 4.2. Second item follows from Theorem 3.4 combined with Theorem 4.2. $\mathrm{c}$

We emphasize that it is NP-hard to compute the treelength of a graph [28], but there exist 3-approximation algorithms to compute it in polynomial-time [20]. Moreover, a longest isometric cycle in a graph can also be computed in polynomialtime [27]. Hence, the previous result gives a new way to compute lower-bounds for treewidth.

4.2. Lower-bound in case of bounded-genus graphs. In this section, we prove that the treewidth of a graph is upper-bounded by a function of its treelength and of its genus. Our result is mainly based on the result from [16] stating that any graph with large treewidth and genus contains a large "grid-like" graph as a contraction. We use their terminology.

Let us remind that a planar triangulation of a planar graph $G$ is a planar supergraph of $G$ whose faces are bounded by triangles. A partially triangulated $(r \times r)$-grid is any graph that contains an $(r \times r)$-grid as a subgraph and is a subgraph of some planar triangulation of the same $(r \times r)$-grid. A $(r, k)$-gridoid $G$ is a partially triangulated $(r \times r)$-grid in which $k$ extra edges have been added ${ }^{5}$.

THEOREM 4.4. [16] Let $G$ be a graph with genus $g$ and $t w(G)>4 k(g+1)$ with $k \geq 12 g$, then $G$ contains a $(k-12 g, g)$-gridoid as a contraction.

We prove that such a gridoid has large treelength and so, since the treelength is contraction-closed, such a graph has large treelength too.

LEMmA 4.5. Let $G$ be a partially triangulated $(r \times r)$-grid, then $t l(G) \geq\lfloor r / 3\rfloor-1$.

\footnotetext{
${ }^{5}$ Note that the notion of $(r, k)$-gridoid is more general in [16].
} 
Proof. The result holds if $r \leq 3$ because in such a case $t l(G) \geq 1 \geq\lfloor r / 3\rfloor-1$. Else, let $G^{\prime}$ be the $(r \times r)$-grid from which $G$ is obtained by planar triangulation. Let $V^{\prime}$ be the set of vertices that are at distance at least $\left\lfloor\frac{r-1}{3}\right\rfloor$ from the external face of $G^{\prime}$. The vertices of $V^{\prime}$ induce a partially triangulated $\left(r^{\prime} \times r^{\prime}\right)$-grid $F$ in $G$, $r=2\left\lfloor\frac{r-1}{3}\right\rfloor+r^{\prime}$, such that the external face has not been triangulated. Moreover, $F$ is isometric in $G$. Hence, $\operatorname{tl}(G) \geq t l(F)$. We show that $t l(F) \geq\lfloor r / 3\rfloor-1$.

Our proof adapts from the lower-bound techniques in $[20$, Sec. 2.3$]$. Let $(T, \mathcal{X})$ be any tree-decomposition of $F$. Consider the two subsets of vertices $A, B$ that contain the first and the last row of $F$ respectively. Since $A$ induces a connected subgraph of $F$, by the properties of tree-decompositions the bags in $\mathcal{X}$ that intersect $A$ form a subtree $T_{A}$ of $T$. Similarly, the bags in $\mathcal{X}$ that intersect $B$ form a subtree $T_{B}$ of $T$. Furthermore, either $T_{A} \cap T_{B} \neq \emptyset$ (in which case, the diameter of every bag in $T_{A} \cap T_{B}$ is at least $\left.r^{\prime}-1\right)$, or by [20, Lemma 5] there exists a bag which intersects all paths between $A$ and $B$ in $F$. In the latter case, such bag must intersect the first and last column of $F$, and so, it has diameter at least $r^{\prime}-1$. Therefore, $(T, \mathcal{X})$ has length at least $r^{\prime}-1$ in both cases, that proves that $t l(F) \geq r^{\prime}-1 \geq\lfloor r / 3\rfloor-1$. $\square$

Lemma 4.6. Let $G$ be a $(r, k)$-gridoid, then $t l(G)>r /(18 \sqrt{2 k+1})-2$.

Proof. The result holds if $r \leq 36 \sqrt{2 k+1}$ because in such case $t l(G) \geq 1>$ $r /(18 \sqrt{2 k+1})-2$. Hence, let us assume that $r>36 \sqrt{2 k+1}$.

Let $M$ be a set of at most $k$ edges whose removal in $G$ yields a partially triangulated $(r \times r)$-grid. Let $S=V(M)$ be the set of end-vertices of the edges of $M$. Note that $|S| \leq 2 k$. Also, let $G^{\prime}$ be the $(r \times r)$-grid whose $G \backslash M$ is a partial planar triangulation. Let finally $4 \leq x \leq r$ be an integer. There are $(r-x+1)^{2}$ distinct $(x \times x)$-grids as subgraphs in $G^{\prime}$, that give us as many distinct partially triangulated $(x \times x)$-grids as subgraphs in $G$. Furthermore, each node in $S$ belongs to at most $x^{2}$ such subgraphs. Therefore assuming $(r-x+1)^{2}-2 k \cdot x^{2} \geq 1$, there is one of these partially triangulated $(x \times x)$-grids, say $H$, that does not contain any node incident to one of the $k$ extra edges. Consider the partially triangulated $\left(x^{\prime} \times x^{\prime}\right)$-grid $R$ which is in the center of $H$, with $x=2 \cdot\left\lfloor\frac{x-1}{3}\right\rfloor+x^{\prime}$. That is, $R$ is a subgraph of $H$ and any node of $R$ is at distance at least $\left\lfloor\frac{x-1}{3}\right\rfloor$ from a node of $G \backslash H$ (it is possible because $H$ does not contain an extremity of an extra edge). Therefore, $R$ is isometric in $G$ and $t l(R) \leq t l(G)$. By Lemma 4.5,

$$
t l(R) \geq\left\lfloor x^{\prime} / 3\right\rfloor-1 \geq x / 9-1
$$

It remains to maximize $x$ satisfying the inequality $(r-x+1)^{2}-2 k \cdot x^{2} \geq 1$ so that we maximize the above lower-bound for $t l(R)$. The polynomial

$$
\begin{aligned}
(r-X+1)^{2}-2 k \cdot X^{2}-1 & =r^{2}+X^{2}+1-2 r \cdot X+2 r-2 X-2 k \cdot X^{2}-1 \\
& =-\left[(2 k-1) \cdot X^{2}+2(r+1) \cdot X-r(r+2)\right]
\end{aligned}
$$

has for reduced discriminant $(r+1)^{2}+r(r+2)(2 k-1)=2 k \cdot r(r+2)+1$, hence its roots are equal to

$$
\left\{-\frac{\sqrt{2 k \cdot r(r+2)+1}+r+1}{2 k-1}, \frac{\sqrt{2 k \cdot r(r+2)+1}-r-1}{2 k-1}\right\} .
$$

Since this polynomial is nonnegative only between its roots, the value maximizing $x$ 
is:

$$
\begin{aligned}
x_{0} & =\left\lfloor\frac{\sqrt{2 k \cdot r(r+2)+1}-r-1}{2 k-1}\right\rfloor \geq \frac{\sqrt{2 k \cdot r(r+2)+1}-r-1}{2 k-1}-1+\frac{1}{2 k-1} \\
& =\frac{r(r+2)}{\sqrt{2 k \cdot r(r+2)+1}+r+1}-1+\frac{1}{2 k-1}>\frac{r(r+2)}{2 \sqrt{2 k \cdot r(r+2)+1}}-1 \\
& >\frac{1}{2} \sqrt{\frac{r(r+2)}{2 k+1}}-1>\frac{r}{2 \sqrt{2 k+1}}-1 .
\end{aligned}
$$

Hence, $\operatorname{tl}(G) \geq t l(R) \geq x_{0} / 9-1 \geq r /(18 \sqrt{2 k+1})-2$. $\square$

THEOREM 4.7. Let $G$ be a graph with genus $g$ and $t w(G)>4 k(g+1)$ with $k \geq 12 g$. Then

$$
t w(G) \leq 72 \sqrt{2}(g+1)^{\frac{3}{2}} \cdot t l(G)+\mathcal{O}\left(g^{2}\right)
$$

Proof. By Theorem 4.4, $G$ contains a $(k-12 g, g)$-gridoid $R$ as a contraction. By Lemma 4.6,

$$
t l(R)>\frac{k-12 g}{18 \sqrt{2 g+1}}-2
$$

Thus, by setting $k=(t w(G)-1) /(4(g+1))$, we obtain that:

$$
t l(R)>\frac{t w(G)-48 g(g+1)-1}{72(g+1) \sqrt{2 g+1}}-2>\frac{t w(G)}{72 \sqrt{2}(g+1)^{\frac{3}{2}}}-\frac{\sqrt{2}}{3} \cdot \sqrt{g+1}-3 .
$$

The result then follows from the fact that treelength is contraction-closed. $\square$

Extensions.. Theorem 4.7 can be extended to the broader class of apex-minorfree graphs. An apex graph is a graph such that the removal of one vertex creates a planar graph. Similar techniques from the bidimensionality theory allow us to deal with graphs that exclude a fixed apex graph as a minor. Namely, we will make use of the graph $\Gamma_{k}$ as it is defined in [23]. The graph $\Gamma_{k}$ is obtained from a $(k \times k)$-grid by triangulating its internal faces such that all internal vertices become of degree 6 , all non-corner external vertices are of degree 4, and then one corner of degree two is joined by edges with all vertices of the external face.

THEOREM 4.8. [23] For every apex graph $H$, there is a constant $c_{H}>0$ such that every connected $H$-minor-free graph of treewidth at least $c_{H} \cdot k$ contains $\Gamma_{k}$ as a contraction.

THEOREM 4.9. Let $H$ be any apex graph and $G$ be a connected $H$-minor-free graph of treewidth at least $c_{H} \cdot k$, where $c_{H}$ is the constant of Theorem 4.8. Then $t l(G) \geq t w(G) /\left(3 \cdot c_{H}\right)-1$.

Proof. By Theorem 4.8, $G$ contains $\Gamma_{k}$ as a contraction. Moreover, $\Gamma_{k}$ is a partially triangulated grid. The result follows from Lemma 4.5 and the fact that treelength is contraction-closed. $\mathrm{C}$

5. Conclusion. We can deduce from Corollary 3.5 and Theorem 4.7 that for every $n$-node graph of genus $g$, the 3 -approximation algorithms for treelength in [20] compute in $\mathcal{O}\left(g \cdot n^{2}\right)$-time an integer $t^{*}$ satisfying:

$$
\frac{t w(G)}{72 \sqrt{2}(g+1)^{\frac{3}{2}}}-\frac{\sqrt{2}}{3} \cdot \sqrt{g+1}-3 \leq t^{*} \leq 3\lfloor\ell(G) / 2\rfloor \cdot t w(G) .
$$


Observe that in case an upper-bound on the treewidth is given, we can also deduce from our relations a lower-bound on the graph genus.

The main drawback with our above approximation algorithm for treewidth is that it may output a tree-decomposition with unbounded width (the length is upper-bounded by $\left.t^{*}\right)$. We let open whether our method can be modified so that it outputs a treedecomposition of width $\mathcal{O}\left(\ell(G) \cdot(g+1)^{3 / 2} \cdot t^{*}\right)$.

Acknowledgments. We wish to thank the referees for their careful reading of the first version of this manuscript, and their useful comments. Their remarks and suggestions have improved the presentation of this paper significantly.

\section{REFERENCES}

[1] Muad Abu-Ata and Feodor F. Dragan, Metric tree-like structures in real-world networks: an empirical study, Networks, 67 (2016), pp. 49-68.

[2] Aaron B. Adcock, Blair D. Sullivan, and Michael W. Mahoney, Tree decompositions and social graphs, arXiv preprint arXiv:1411.1546, (2014).

[3] Stefan Arnborg, Derek G. Corneil, and Andrzej Proskurowski, Complexity of finding embeddings in a k-tree, SIAM J. Algebraic Discrete Methods, 8 (1987), pp. 277-284.

[4] Hans-JÜrgen Bandelt And Victor Chepoi, Metric graph theory and geometry: a survey, Contemporary Mathematics, 453 (2008), pp. 49-86.

[5] Rémy Belmonte, Fedor V. Fomin, Petr A. Golovach, and M. S. Ramanujan, Metric dimension of bounded width graphs, in Mathematical Foundations of Computer Science 2015: 40th International Symposium, MFCS 2015, Milan, Italy, August 24-28, 2015, Proceedings, Part II, 2015, pp. 115-126.

[6] Hans L. Bodlaender, A partial k-arboretum of graphs with bounded treewidth, Theor. Comput. Sci., 209 (1998), pp. 1-45.

[7] Marián Boguna, Fragkiskos Papadopoulos, and Dmitri Krioukov, Sustaining the Internet with hyperbolic mapping, Nature Communications, 1 (2010), pp. 1-18.

[8] Jérémie Chalopin, Victor Chepoi, Hiroshi Hirai, and Damian Osajda, Weakly modular graphs and nonpositive curvature, arXiv preprint arXiv:1409.3892, (2014).

[9] Victor Chepoi, Feodor Dragan, Bertrand Estellon, Michel Habib, and Yann Vaxès, Diameters, centers, and approximating trees of $\delta$-hyperbolic geodesic spaces and graphs, in Proceedings of the twenty-fourth annual symposium on Computational geometry, ACM, 2008, pp. 59-68.

[10] Victor Chepoi and Bertrand Estellon, Packing and covering $\delta$-hyperbolic spaces by balls, in Approximation, Randomization, and Combinatorial Optimization. Algorithms and Techniques, vol. 4627 of Lecture Notes in Computer Science, Springer, 2007, pp. 59-73.

[11] David M Chickering, Dan Geiger, and David Heckerman, On finding a cycle basis with a shortest maximal cycle, Information Processing Letters, 54 (1995), pp. 55-58.

[12] David Coudert, Guillaume Ducoffe, and Nicolas Nisse, Diameter of Minimal Separators in Graphs, Research Report RR-8639, Inria Sophia Antipolis ; I3S, nov 2014. https://hal.inria.fr/hal-01088423.

[13] Bruno Courcelle, The monadic second-order logic of graphs. I. recognizable sets of finite graphs, Information and Computation, 85 (1990), pp. $12-75$.

[14] Fabien de Montgolfier, Mauricio Soto, and Laurent Viennot, Treewidth and hyperbolicity of the internet, in 10th IEEE International Symposium on Network Computing and Applications (NCA), Boston, 2011, IEEE, pp. 25-32.

[15] Erik D. Demaine and Mohammad Taghi Hajiaghayi, The bidimensionality theory and its algorithmic applications, Comput. J., 51 (2008), pp. 292-302.

[16] Erik D. Demaine, Mohammad Taghi Hajiaghayi, and Dimitrios M. Thilikos, The bidimensional theory of bounded-genus graphs, SIAM J. Discrete Math., 20 (2006), pp. 357-371.

[17] Youssou Dieng and Cyril Gavollle, On the tree-width of planar graphs, Electronic Notes in Discrete Mathematics, 34 (2009), pp. 593-596.

[18] Reinhard Diestel, Graph theory, Heidelberg, Graduate Texts in Mathematics, 173 (2010), p. 451 pp. $4 t h$ edition.

[19] Reinhard Diestel and Malte Müller, Connected tree-width, nov 2014. http://arxiv.org/abs/1211.7353. 
[20] Yon Dourisboure and Cyril Gavollle, Tree-decompositions with bags of small diameter, Discrete Mathematics, 307 (2007), pp. 2008-2029.

[21] Pierre Duchet, Michel las Vergnas, and Henry Meyniel, Connected cutsets of a graph and triangle bases of the cycle space, Discrete Mathematics, 62 (1986), pp. 145-154.

[22] Uriel Feige, MohammadTaghi Hajiaghayi, and James R. Lee, Improved approximation algorithms for minimum weight vertex separators, SIAM J. Comput., 38 (2008), pp. 629657.

[23] Fedor V. Fomin, Petr A. Golovach, and Dimitrios M. Thilikos, Contraction obstructions for treewidth, J. Comb. Theory, Ser. B, 101 (2011), pp. 302-314.

[24] Walter Hohberg, The decomposition of graphs into k-connected components, Discrete mathematics, 109 (1992), pp. 133-145.

[25] John D. Horton, A polynomial-time algorithm to find the shortest cycle basis of a graph, SIAM Journal on Computing, 16 (1987), pp. 358-366.

[26] Robert Krauthgamer and James R. Lee, Algorithms on negatively curved spaces, in Foundations of Computer Science, 2006. FOCS'06. 47th Annual IEEE Symposium on, IEEE, 2006, pp. 119-132.

[27] Daniel Lokshtanov, Finding the longest isometric cycle in a graph, Discrete Applied Mathematics, 157 (2009), pp. 2670-2674.

[28] - On the complexity of computing treelength, Discrete Applied Mathematics, 158 (2010), pp. 820-827.

[29] Richard Nowakowski and Peter Winkler, Vertex-to-vertex pursuit in a graph, Discrete Mathematics, 43 (1983), pp. 235-239.

[30] Andrea Parra and P. Scheffler, Characterizations and algorithmic applications of chordal graph embeddings, Discrete Applied Mathematics, 79 (1997), pp. 171-188.

[31] Neil Robertson and Paul D. Seymour, Graph minors. II. algorithmic aspects of tree-width, Journal of Algorithms, 7 (1986), pp. 309-322. 